\title{
Application of a new integrated landscape index to predict potential urban heat islands
}

\author{
Ailian Chen ${ }^{\mathrm{a}, \mathrm{b}}$, Xinfeng Zhao ${ }^{\mathrm{c}}$, Lei Yao ${ }^{\mathrm{a}}$, Liding Chen ${ }^{\mathrm{a}, *}$ \\ a State Key Lab of Urban Regional Ecology, Research Center for Eco-Environmental Sciences, Chinese Academy of Sciences(CAS), Beijing 100085, PR China \\ ${ }^{\mathrm{b}}$ Chinese Academy of Agricultural Engineering, Beijing 100125, PR China \\ ' Digital Agriculture E Global Disasters Division, Institute of Remote Sensing and Digital Earth, CAS, 100094 Beijing, PR China
}

\section{A R T I C L E I N F O}

\section{Article history:}

Received 20 January 2016

Received in revised form 6 April 2016

Accepted 24 May 2016

Available online 10 June 2016

\section{Keywords:}

Landscape pattern

LST

Location weighted landscape index

LWLI

Urbanization

\begin{abstract}
A B S T R A C T
Studies on the interactions between urban landscape patterns and land surface temperatures are the key to urban heat island (UHI) mitigation. However, the landscape pattern indices used in previous studies were inconsistent in type and number. Furthermore, few studies combined the composition and configuration indices into one integrated indicator. The description of landscape pattern is thus a great yet rewarding challenge. In this study, we used the integrated location weighted landscape index (LWLI) proposed by Chen et al. (2009) and revised it to indicate potential UHIs. Fifty-six circular landscape samples along four transects were created and the LWLI was derived based on the theory of the GINI index. The LWLI considers the type, composition and configuration of different land covers inside a landscape, where configuration is treated as location weights. The potential UHI was represented by the land surface temperature (LST) of each circle landscape center. The correlation analysis results showed that the LWLI was significantly positively correlated with summer potential UHIs, with a Pearson R equaling 0.736 , and barely correlated with winter potential UHI. Moreover, the LWLI was slightly more strongly correlated with potential UHI than composition alone. These correlations weakened as the landscapes grew larger. The linear regression results further revealed that the LWLI explained about $53 \%$ of the summer potential UHI, which was slightly better than composition alone (49\%). This indicated that the LWLI was as effective in predicting the potential UHI as the combination of several pattern indicators, echoing Tobler's first law of geography, which states that "all things are related, but nearby things are more related than distant things." This study also leaves room for improvement of the index by integrating more environmental/ecological parameters as weights, and for further application of the index in other fields.
\end{abstract}

(c) 2016 Elsevier Ltd. All rights reserved.

\section{Introduction}

Global urbanization often leads to the deterioration of the urban climates, causing urban heat islands (UHIs). Originally, and still most commonly, the term UHI refers to the phenomenon that more urbanized areas have a higher air temperature than their surrounding suburban areas. With the development of thermal infrared remote sensing in the last few decades, UHI can also refer to surface UHI; namely, the difference in land surface temperature (LST) at the urban canopy layer or the urban boundary layer (Buyantuyev and Wu, 2010; Saaroni et al., 2000; Voogt, 2002). The LST is the

\footnotetext{
* Corresponding author.

E-mail addresses: cal-0601@163.com (A. Chen), zhaoxf@radi.ac.cn (X. Zhao), alex_yaolei@163.com (L. Yao), Liding@rcees.ac.cn (L. Chen).
}

mean radiative skin temperature, determined by air temperature and long-wave radiation between the surface and the atmosphere (Nichol et al., 2009; Weng, 2009). Unlike air temperature, LST has a higher spatial resolution and relates more closely to surface land use or landscape pattern, which can easily be derived from remote sensing data (Imhoff et al., 2010; Voogt and Oke, 2003; Weng, 2009). Thus, surface UHI and its associated data source, LST, have been increasingly used in the study of urban climate in recent decades (EPA, 2009; Li et al., 2013; Mirzaei and Haghighat, 2010). Previous studies have shown that both air and surface UHIs are associated with heat waves (Changnon et al., 1996; Fischer et al., 2007), disease (Liu and Weng, 2009) and pose a health risk to more than $50 \%$ of the world's population (Frumkin and McMichael, 2008; $\mathrm{Wu}, 2010)$. Hence, sustainable urbanization is urgently needed to 
mitigate the effects of UHIs and improve urban living conditions (Opdam et al., 2009; Wu, 2010).

For this purpose, a large body of research (Chen et al., 2014; Connors et al., 2013; Li et al., 2011; Weng et al., 2007; Zhou et al., 2011) has focused on the relationship between surface UHIs and landscape patterns using landscape indices based on the concept of landscape ecology; that is, the theory that landscape patterns strongly affect and are affected by ecological processes (Turner, 1989). Composition and configuration, as two aspects of landscape patterns, were both tested (Chen et al., 2014; Connors et al., 2013; Zhou et al., 2011), with the conclusion that composition is more effective than configuration in influencing LSTs or surface urban heat islands (SUHIs) (Chen et al., 2014; Li et al., 2012; Zhou et al., 2011).

However, there has been less consistency in the type and number of landscape indices used in previous studies (Chen et al., 2014). Usually, a set of metrics rather than an integrated metric was needed to describe the landscape pattern. The percentage of a certain class in the whole landscape (PLAND) as a composition metric is a prerequisite and is the most used (Chen et al., 2014; Connors et al., 2013; Zhou et al., 2011), whereas several other configuration metrics were also needed, including shape metrics, edge metrics and isolation metrics. For instance, the perimeter-area ratio(PARA), shape index (SI) and fraction index (FRAC) are used to describe shape while the gyration index (GYRATE), total edge (TE), edge density (ED) and so on are used to describe the edge (McGarigal et al., 2012). One or several shape, edge and diversity metrics were always added to PLAND to indicate SUHIs.

Numerous metrics (Baker and Cai, 1992; McGarigal and Marks, 1994; McGarigal et al., 2002, 2009; O'neill et al., 1988) have been proposed and used to describe landscape patterns, and landscape composition and configuration have been addressed. These metrics are not only used in UHI-landscape pattern analysis, but also in many other fields such as water quality and biodiversity (Schindler et al., 2013; Uuemaa et al., 2013). Nonetheless, these metrics face serious problems with redundancy and difficulty in interpretation (Cushman et al., 2008; Kupfer, 2012; Uuemaa et al., 2013). Chen et al. (2014) showed that landscape metrics exhibit high redundancy in indicating LSTs, and that proportion is the most important factor affecting LSTs.

Recently, there have been some appreciable efforts to create indicators linking landscape patterns to ecological function (Chen et al., 2009; Ku'ulei et al., 2012). This is a good sign and a necessary trend, especially when combined with spatial analysis (Kupfer, 2012). Ku'ulei et al. (2012) considered the classes and composition of a landscape, whereas Chen et al. (2009) also considered landscape configuration, and constructed an integrated landscape index they named the location-weighted landscape index (LWLI). The theoretical basis of the LWLI, is "source-sink" landscape theory (Chen et al., 2008, 2009) and Tobler's first law of geography (1970), which states that "all things are related, but nearby things are more related than distant things." "Source-sink" landscape theory (Chen et al., 2008) classifies the attribute class within a landscape as "source," "sink" or "flow," according to its contribution to the targeted ecological process. The construction of the LWLI falls within the frameworks of the Lorenz Curve and the GINI index (Chen et al., 2009; Gastwirth, 1972). One trial, in which the LWLI and "sourcesink" landscape theory were used to assess the effects of landscape type and configuration on nutrient losses in a watershed (Chen et al., 2009) proved to be both efficient and effective.

In this study, we used the LWLI to describe landscape patterns in urban areas to examine its performance in predicting UHIs. Tobler's first law of geography implies that the LST of a landscape center would be more affected by the nearer landscape, and urban areas tend to form a heat center with more "hot" land acting as a heat "source" located around the center. Consequently, the hypothesis is that the LWLI describing the distribution of "source" and "sink" land cover around a landscape center might be correlated with the center LST. The subsequent analysis focused on testing this hypothesis to find the seasonal performances of the LWLI in indicating the center LST in different scales of landscape. The index is meant to be useful in describing the landscape structure around an urban center, and is helpful in predicting the potential of a UHI effect for an urban planning graph.

\section{Methodology}

\subsection{Study area and sample landscapes}

We took Beijing $\left(39.9^{\circ} \mathrm{N}, 116.3^{\circ} \mathrm{E}\right)$, China as the case study area. Beijing has a monsoon-influenced humid continental climate characterized by hot-humid summers, due to the East Asian monsoon, and cold-windy-dry winters, due to the Siberian anticyclone. The highest average daytime temperature in January is around $1^{\circ} \mathrm{C}$, whereas in July the highest average daytime temperature is higher than $30^{\circ} \mathrm{C}$. Although the average UHI's summer daytime air intensity in the 1970 s was only about $1{ }^{\circ} \mathrm{C}$, and has only increased $0.31^{\circ} \mathrm{C}$ every ten years since then (Yu et al., 2005), the surface UHI analysis shows a higher UHI intensity, ranging from 5 to $10^{\circ} \mathrm{C}$ (Wang et al., 2007).

The city spreads out in five concentric ring roads, which go from the Second Ring Road around the urban center to the Sixth Ring Road in the suburbs, as shown in Fig. 1. The region within the Fifth Ring Road was used as the study area because it is the main metropolitan area.

Four transects were created and points were assigned on them at $1 \mathrm{~km}$ intervals. This resulted in 56 sample points, as shown in Fig. 1. Round sample landscapes were created based on these points by the Buffer Analysis Tool in the ArcGIS ${ }^{\mathrm{TM}}$ software. Four radii-500, 1000,1500 and $2000 \mathrm{~m}$-were used to derive the round buffers representing different scales of circular landscapes.

\subsection{Materials}

\subsubsection{Land cover map}

Six swaths of QuickBird (QB) images were used to obtain land cover data. The central parts of the study area $(\sim 75 \%)$ were covered by two QB images taken on July 5, 2002. The other parts were covered by four QB images, with the two eastern images taken on April 24, 2002 and the two western images taken on April 1, 2002.

Unsupervised classification and the decision tree method were combined to obtain the land cover map, as detailed in Chen et al. (2014). Four types of land cover were considered for land use classification: vegetation, water, bare soil and built up areas or impervious surfaces (ISs). The vegetation in the metropolis was mainly a mix of grass, shrubs and trees. ISs included buildings and impervious roads. Bare soil was generally bare land. "Water" referred to rivers and lakes.

The overall accuracy was $85.2 \%$ (31674/37174 pixels), with a Kappa coefficient of 0.79 . The mapping resulted in a pattern of land cover with 931.0 ha (1.4\%) of water, 26882.5 ha (40.3\%) of vegetation and 38915.3 ha (50.3\%) of built up surface, plus bare soil ( $8.0 \%)$, as shown in Fig. 2.

Please note that $4.16 \%$ of the central parts of the QB images are clouds. We used the land cover date mapping from the IKONOS images on July 9, 2012 to replace the cloud area. The cloudy parts were mostly located in the already urbanized area, where less land cover was changing. Moreover, we used the transect samples, which cover only small sections of the clouds. The error from cloud effects was thus negligible. 

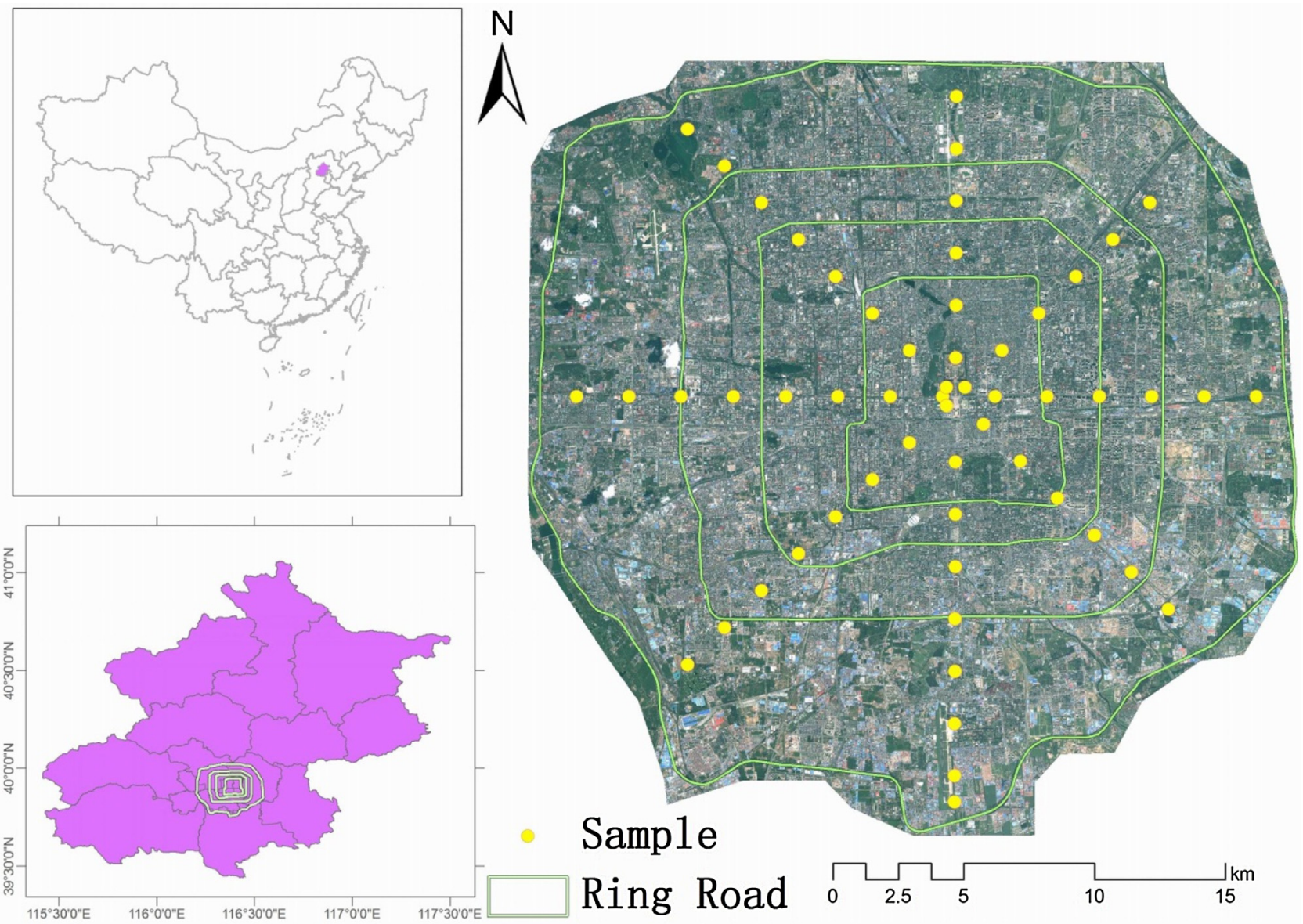

Fig. 1. Location of the study area and the samples used for research.

\subsubsection{Land surface temperature}

One swath of the ETM+ image covers the whole study area. Four swaths of cloud-free Landsat ETM+ images were selected to retrieve LSTs, dated March 19, May 22, July 9 and October 13, 2002. March 19 and October 13 represented the dry and cool season, whereas May 22 and July 9 represented the hot and humid season.

The ETM+ images were geometrically rectified and registered to the same projection system (datum: WGS 84, UTM Zone North 50) as all of the QuickBird images in the ENVI ${ }^{\mathrm{TM}}$ software. Band 6 of the $\mathrm{ETM}+$ data was resampled to a resolution of $30 \mathrm{~m}$, as were Bands 3 and 4, and the former was used to retrieve the LST.

The LST data were obtained by three steps, as detailed in Chen et al. (2014) and Jiménez-Muñoz and Sobrino (2003): (1) the digital number was converted to at-sensor radiance with the scaling parameters provided along with the image metafile (NASA, 2000), (2) the at-sensor radiance was converted to at-sensor temperature and (3) the at-sensor temperature was adjusted to LST with land surface emissivity $(\varepsilon)$ determined by normalized difference vegetation index.

The resulting LST in units Kelvin (K) was finally converted to Celsius $\left({ }^{\circ} \mathrm{C}\right)$. The mean LST of the whole area and the four land-cover types on four dates are shown in Table 1.

\subsection{Derivation of the LWLI}

The LWLI was once proposed to describe the landscape pattern of a watershed towards the water outlet, and was related to pointbased measurements of nutrient losses or soil erosion. It was built within the frameworks of the Lorenz curve and the GINI index
Table 1

Mean LST of each type of land cover and the whole study area on four dates 20020319, 20020522, 20020709 and 20021013 (date format YYYYMMDD).

\begin{tabular}{lllll}
\hline Mean LST $\left({ }^{\circ} \mathrm{C}\right)$ & 20020319 & 20020522 & 20020709 & 20021013 \\
\hline Water & 10.83 & 25.70 & 24.93 & 15.03 \\
Vegetation & 17.52 & 33.60 & 26.69 & 17.91 \\
Bare soil & 19.30 & 40.43 & 28.98 & 20.68 \\
Built up area & 18.75 & 40.59 & 29.80 & 20.68 \\
Whole study area & 17.67 & 37.92 & 28.35 & 19.06 \\
\hline
\end{tabular}

(Chen et al., 2009; Gastwirth, 1972) The Lorenz curves for LWLI were formed with normalized distance from the center as the $\mathrm{X}$ axis, and normalized land cover area as the $Y$ axis. The integral areas were calculated by multiplying the normalized area with the normalized distance, indicating the structure/configuration of the land cover type towards the water outlet point. LWLI was calculated according to GINI index by Equation (1).

$\mathrm{LWLI}=\sum_{i}^{m} A-S R C \cdot \int_{0}^{1} N A S S R C / \sum_{i}^{m} A-S R C \cdot \int_{0}^{1} N A S R R+\sum_{i}^{m} A-S N K \cdot \int_{0}^{1} N A S S N$

where ASRC is the area of the "source" land cover versus ASNK, which is the area of the "sink" land cover within a watershed. NA_SRC and NA_SNK are the normalized area of a "source" or "sink" class to the whole landscape area. Thus, the higher integral means that there is proportionally more land cover near the outlet, and vice versa. 


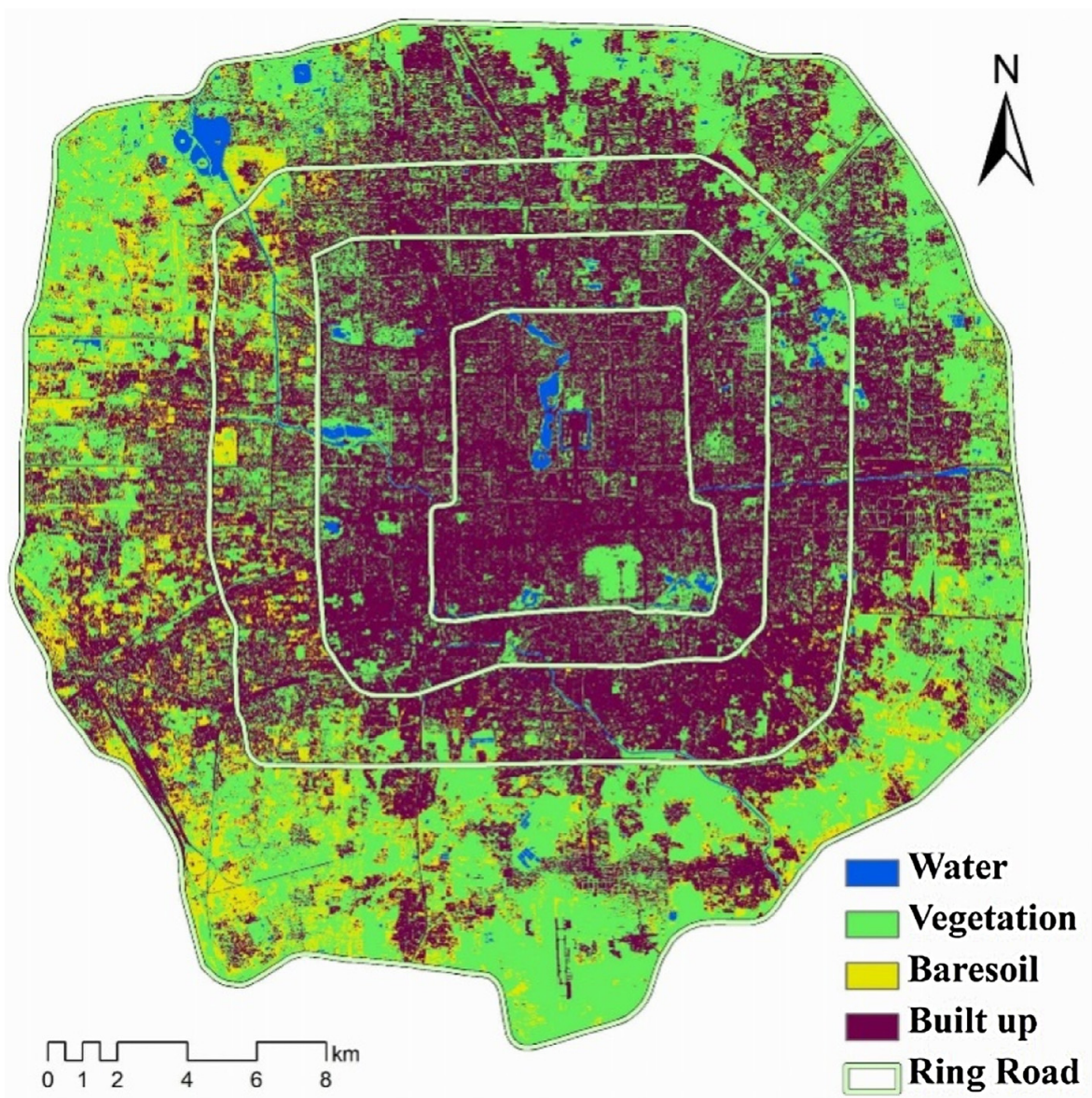

Fig. 2. Land cover map.

The LWLI considers the effect of land cover type along with its composition and configuration. The configuration is mainly the normalized area of each land cover weighted by the location relative to the water outlet point.

As for UHI and for thermal radiation, the function between landscape patches is spherical. When a certain landscape forms a heat island, it is the result of the island itself along with the surrounding radiation. Therefore, we took circular landscape sample to study the effect of surrounding land cover towards the landscape center.

The bare soil and built up area had higher LSTs and were considered "sources" for the potential UHI, whereas water and vegetation were considered "sinks" for potential UHI. For each landscape, four LWLIs with four scales were calculated based on four types of circle landscapes with different radii, i.e. 500, 1000, 1500 and $2000 \mathrm{~m}$. The LWLI was built according to the following 3 steps.

First, ring buffers with $100 \mathrm{~m}$ intervals were created for each circle landscape sample, as shown in Fig. 3a and c. The distances were normalized to a fractional part between 0 and 1 . For instance, the derivation of the $\mathrm{LWLI}$ for the landscape with a $1000 \mathrm{~m}$ radius normalized the distances to landscape center between 0 and 1 , with 0.1 as the interval. For the $500 \mathrm{~m}$ landscapes, the normalized buffer interval was 0.2 , for the $1500 \mathrm{~m}$, it was 0.667 and for the $2000 \mathrm{~m}$ circle landscape, it was 0.05 .

Second, the area of a certain land cover type within each buffer interval was normalized by the total area of the class within the landscape, and was increased from 0 to 1 as the buffer interval increased.

ArcGIS $^{\mathrm{TM}}$ software was used to create buffers and for the area normalization of all of the landscape cover. MATLAB was used to accumulate the Lorenz curve and calculate the LWLI. Two examples of the Lorenz curve and the LWLI deviation values are shown in Figs. 3, with Fig. 3a and c showing the landscape sample, whereas Fig. $3 \mathrm{~b}$ and d giving the corresponding LWLIs.

Third, the LWLI was derived using Equation (1), in which the sum of the source area by its structure is divided by the entire class.

\subsection{Statistical analysis}

The normal distributions of the LWLI were tested, and the results showed that all of the LWLIs in the four landscapes were normally distributed. Pearson correlations were then conducted between the LWLIs of different scales and the mean LST of the landscape center for different dates. The mean LST of the landscape center indicating potential UHI was represented by the mean LST of the center circle with $100 \mathrm{~m}$ as its radius.

The proportion(PROP) of built up areas as hot land cover without the combination of configuration was also correlated with the LST to compare the performances of the LWLI and the PROP. The determinative effects of the LWLI to the potential UHIs on the most correlated dates were further explored by unary linear regressions.

\section{Results}

\subsection{Seasonal variations in the relationship between the LWLI and center LST}

The Pearson correlation between the LWLIs of different scales and the center mean LST is shown in Table 2. A significant positive correlation was seen between the center LST of hot dates (July 9, May 22) and the LWLI, and between the center LST and the PROP of 


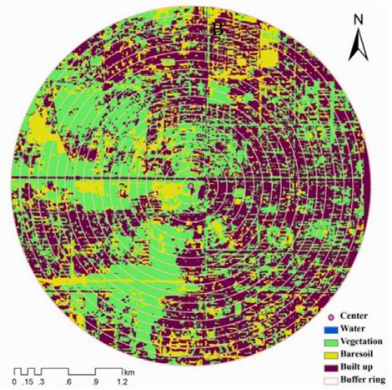

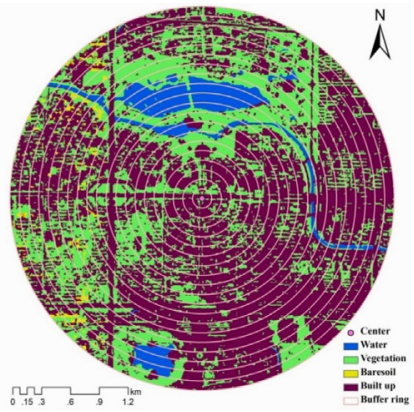

b
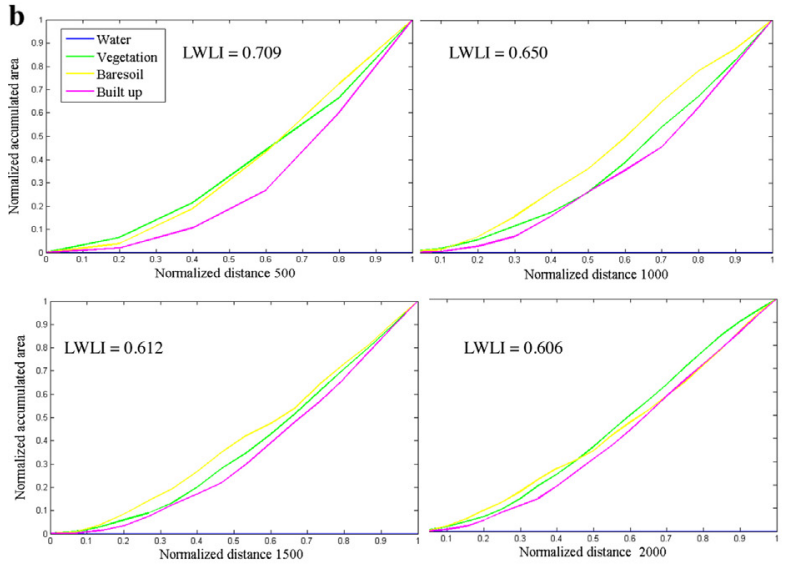

d
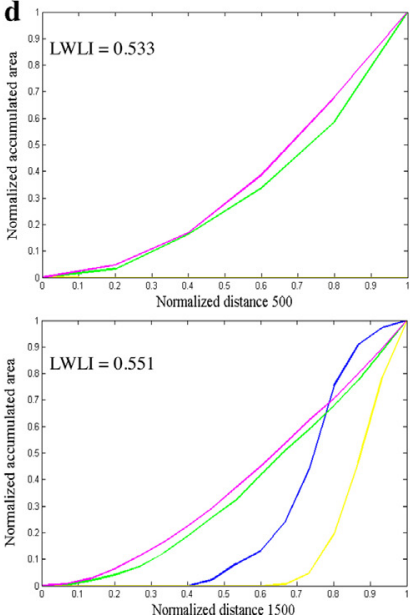
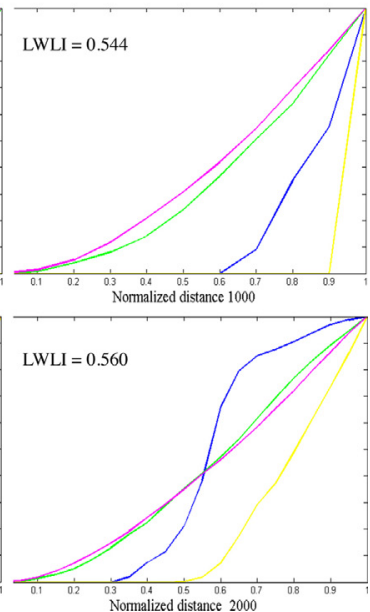

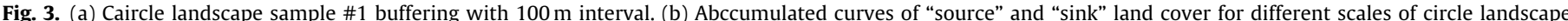

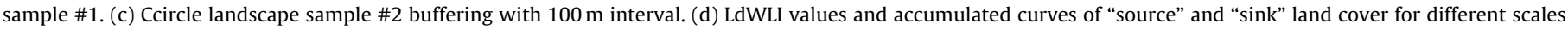
circle landscape sample \#2.

Table 2

Pearson correlation coefficients between seasonal center LST and the LWLIs of different distances. LSTYYYYMMDD is the center mean LST on the dates (given as YYYYMMDD). LWLI_500, LWLI_1000, LWLI_1500 and LWLI_2000 and PROP500, PROP1000, PROP1500 and PROP2000 represent the LWLI and the PROP of hot land cover for four different circle landscape scales.

\begin{tabular}{lllll}
\hline Indicators & LST20020709 & LST20020522 & LST20021013 & LST20020319 \\
\hline LWLI_500 & $0.736^{*}$ & $0.364^{* *}$ & & \\
PROP_500 & $0.701^{* *}$ & $0.316^{*}$ & & \\
LWLI_1000 & $0.722^{* *}$ & $0.321^{*}$ & & \\
PROP_1000 & $0.705^{* *}$ & $0.302^{* *}$ & & \\
LWLI_1500 & $0.708^{* *}$ & $0.304^{*}$ & \\
PROP_1500 & $0.684^{* *}$ & $0.273^{*}$ & \\
LWLI_2000 & $0.696^{* *}$ & $0.280^{*}$ & \\
PROP_2000 & $0.670^{* *}$ & &
\end{tabular}

Note: ${ }^{* *}$ Means that the correlation is significant at the 0.01 level (2-tailed) and ${ }^{*}$ means that the correlation is significant at the 0.05 level (2-tailed).

hot/source land cover. The LWLI has a slightly stronger correlation with the center LST than does the PROP of hot/source land cover. In cooler months such as October and March, no significant correlations were observed between the LST and both the LWLI and the PROP. The correlation coefficients between the LST of the same date and the LWLI decreased as the LWLI scales increased.

\subsection{Determinative effects of the LWLI on the center LST}

The results of the unary linear regressions between the LWLIs of the four scales and the center mean LST are shown in Fig. 4. One scatter plot of the PROP with the mean LST is also shown in Fig. 4. All of the regressions were significant at the 0.01 level. The LWLI of the landscape with $500 \mathrm{~m}$ as its radius had the highest determinative coefficients $\left(R^{2}=0.534\right) . R^{2}$ decreased progressively to 0.474 as the radius of the landscape increased to $2000 \mathrm{~m}$. The LWLI of the landscape with a radius of $500 \mathrm{~m}$ explained $53 \%$ of its center LST, whereas the PROP of the "hot" land cover explained about $49 \%$ of the center LST.

\section{Discussion}

The description of landscape patterns is a great yet rewarding challenge, as they influence and are influenced by ecological processes. In this study, we contribute to landscape pattern analysis by using the LWLI. Although this tool is not perfect, its use provides insights into landscape pattern description, UHI prediction and urban planning.

\subsection{Implications of the LWLI}

The derivation of the LWLI has two useful implications. The first is from the accumulated curves, as described in Section 2.3. The curves illustrate the concentration of "hot" or "source" versus "cool" or "sink" land cover around a landscape center to represent the structure of the whole circle landscape. The second implication is from the LWLI calculation procedure, which combines the structure and proportions of "hot" versus "cool" land cover around a center to predict potential UHIs. The higher the LWLI is, the more concentrated the "hot" land cover is around the center, and thus the higher the center LST. Additionally, the LWLIs of circular landscapes with different radii have different effects on the center LST, in which the LWLI of the small-scale landscape affects the center 

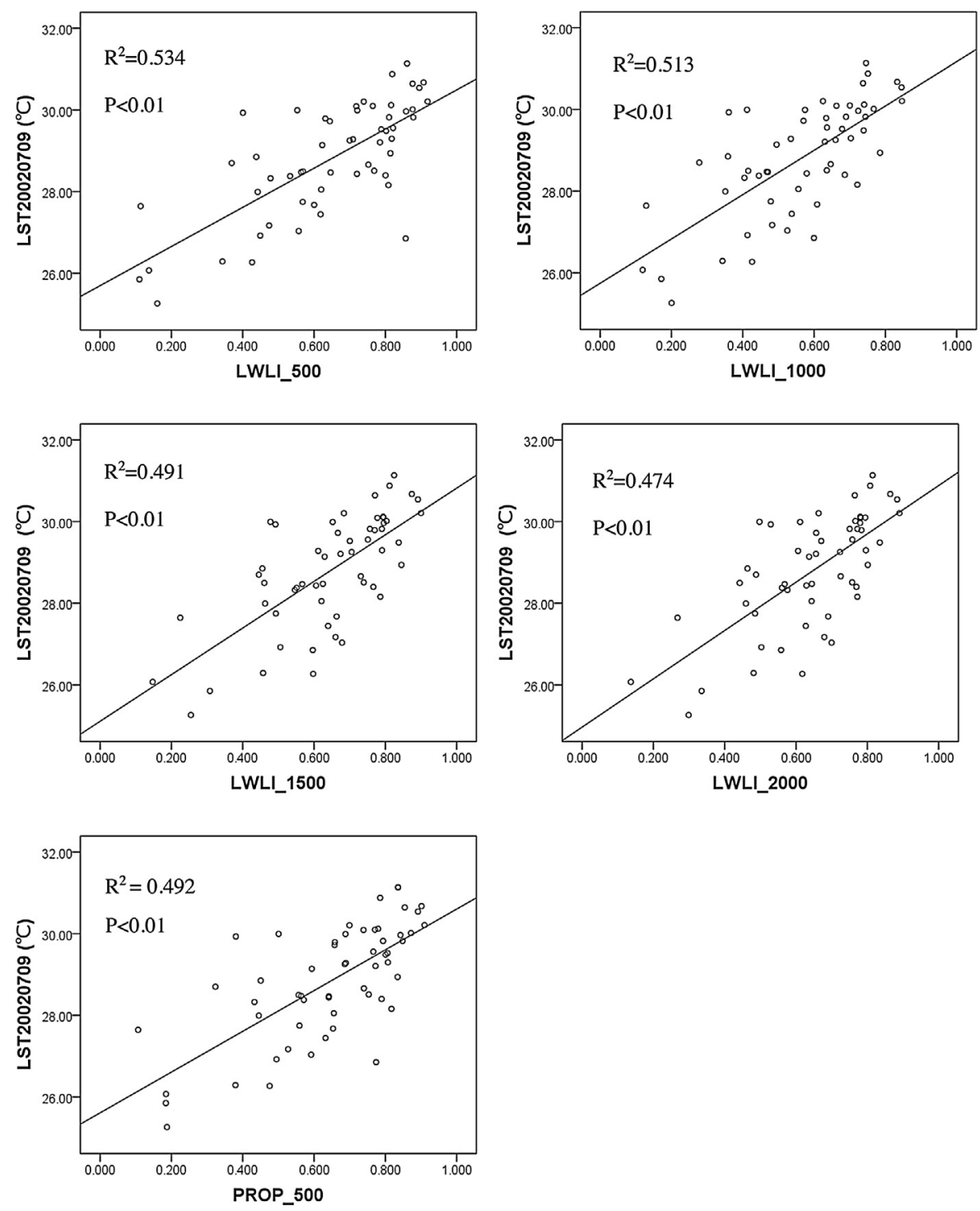

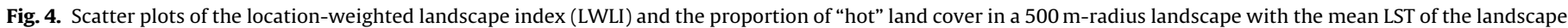
center on four different dates.

LST more strongly. This is in line with Tobler's Law of geography, that "all things are related, but nearby things are more related than distant things."

\subsection{Application of the LWLI}

There are several fields in which the LWLI can be applied. First, the correlation and regression results between the LWLI and the landscape center's mean LST provide some suggestions for urban planning. As the results showed, the composition of land cover plays a more important role in determining LST than the configuration or PROP alone. This implies that the more important factor affecting the LST is the proportion rather than structure or configuration. The regression results further showed that the determinant coefficient of the PROP (composition of hot land cover) explained $49 \%$ of the center mean LST for the 500 m circle landscape, whereas the LWLI explained another $4 \%$. This also proves that structure only matters slightly in predicting the LST. These results are similar to those of previous studies (Zhou et al., 2011), which used many landscape metrics in FRAGSTATS, and echoed the results from Chen et al. (2014), in which composition explained about $49 \%$ of the LST and configuration explained no more than $10 \%$. Thus, the greening of roofs (Oberndorfer et al., 2007) or green walls, especially near a targeted community, that increase the proportion of urban green cover are preferred to redesigning the existing urban green spaces in urban planning.

Second, the LWLI uses categorical maps and can be used to assess a landscape or a planned graph of a block or town center's UHI potential, as most modern planning diagrams are categorical maps.

Finally, the LWLI can be used in general landscape pattern description. It describes the spatial composition and configuration of a certain urban area with one index, instead of using numerous metrics for multiple aspects of landscape patterns. The LWLI successfully combines composition and configuration into one index and can be extended to other ecological processes that relate to Tobler's first law of geography and landscape patterns.

\subsection{Limitations}

As with almost all other landscape indices, the LWLI has limitations, of which the biggest is the data source. The LWLI is designed for categorical maps, rather than for maps of continuous parameters. Thus, the "wall-to-wall" cutting of the land continuous surface (McGarigal et al., 2009) of categorical maps creates problems of 

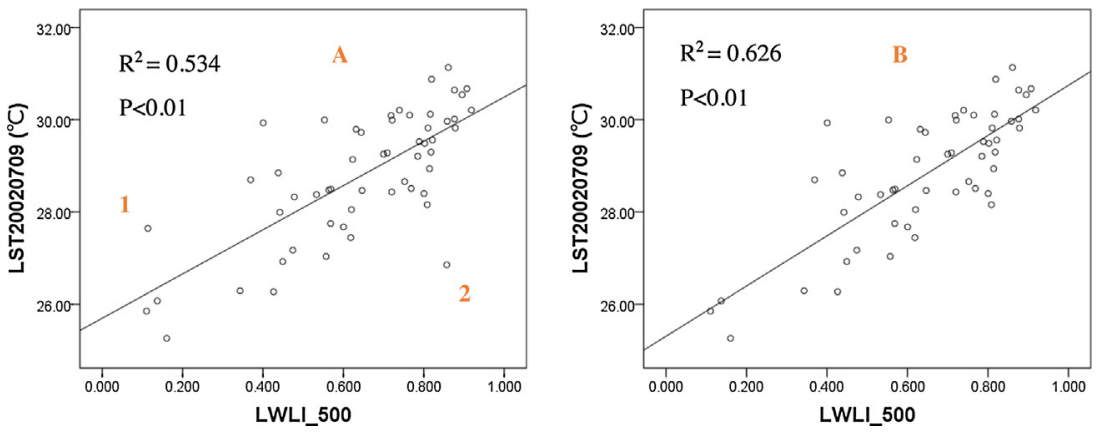

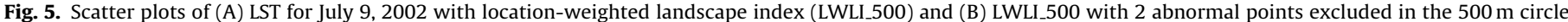
landscape.

generalization and loss of information. In detail, the two abnormal points in Fig. 5(A) are the result of generalization. Point 1 has a lower LWLI but a relatively high LST because the landscape center is close to the airstrips of an airport. The presence of airplanes on the runways might affect the LST. Point 2 has a very high LWLI but a slightly low LST, because it includes a lot of water, which greatly cools the surroundings. Excluding these two points results in a determinative coefficient as 0.626 versus the original of 0.534 , as shown in Fig. 5(B).

Fig. 5 Scatter plots of (A) LST for July 9, 2002 with locationweighted landscape index (LWLI_500) and (B) LWLI500 with 2 abnormal points excluded in the $500 \mathrm{~m}$ circle landscape

In our trial study, bare soil and impervious areas were treated equally for simplification, as were water and vegetation, which actually function differently in magnitude in UHI formation or mitigation. For example, water has been reported to cool down the LST 2.43 times better than vegetation (Liu et al., 2013). The heating rate of bare soil and built up areas are also different. This rate might change in different seasons and different places. Hence, it is important to weight the LWLI with specific and reasonable parameters.

Our study did not add any other weights associated with ecological processes. Some of the thermal features of different land cover types could be tested and added to weight the LWLI instead of simplifying them into the two categories of hot and cool land cover. Our study also does not consider the effects of land use, only land cover. This could be the reason that very recently a study using the moment of inertia of an area of land cover including buildings showed that architecture is more important than composition ( $\mathrm{Li}$ et al., 2016). However, adding weights to an index is also challenging in this discipline due to the complexity of ecological processes. The integration of any physical or thermal parameters that are directly associated with the ecological processes requires further analysis regarding process-related mechanisms. We thus intend to build a more integrative landscape index integrating both landscape patterns and the physical, or in this case, thermal parameters related to urban climate formation to predict UHIs.

\section{Conclusion}

We have now tried our best to strengthen the contribution of our work by linking it to the development of landscape ecology and landscape pattern analysis. The following sentences are added to the beginning of the conclusion section: "Landscape" ecology has become one of the most important fields in the ecology discipline, especially in applied ecology. It is almost a goal for landscape ecology to provide guides for sustainable urbanization or bio-diversity conservation by exploring the relationship between landscape patterns and ecological processes. However, the quantification of landscape patterns is still an obstacle and challenge for not being able to integrating land cover type, composition and configuration inside of a landscape. One reason is the abuse of landscape metrics. This study therefore applied a new integrated landscape index, the LWLI, and revised it to indicate potential UHIs, using the existing software ARCGIS, ENVI and MATLAB. The LWLI combined the effects of land types as well as the two aspects of landscape patterns (composition and the structure or configuration) instead of using many metrics with each one depicting only one aspect of one land cover type. This avoided the problem of redundancy while still performing on par with the combination of many landscape metrics. The LWLI can be used to predict the potential UHI effects for any two-dimension planning graphs. The Lorenz curve and the Gini index were used to describe the distribution of 'hot' towards "cool" land cover around a landscape center, and the first law of geography was echoed, which presents an interesting cross-disciplinary topic. It is still a great challenge to explore new landscape pattern indicators, yet it is by changing landscape patterns that some beneficial ecological processes can be achieved. This study was a first attempt, and it leaves room for further studies.

\section{Acknowledgements}

The authors owe great thanks to the anonymous reviewers for their time and comments. The Landsat ETM+ images are courtesy of the U.S. Geological Survey. The whole work was funded by the Natural Science Foundation of China (41230633; 41590841) and the "High Resolution Earth Observation System" Special Major Projects.

\section{References}

Baker, W.L., Cai, Y., 1992. The r.le programs for multiscale analysis of landscape structure using the GRASS geographical information system. Landscape Ecol. 7 291-302.

Buyantuyev, A., Wu, J., 2010. Urban heat islands and landscape heterogeneity: linking spatiotemporal variations in surface temperatures to land-cover and socioeconomic patterns. Landscape Ecol. 25, 17-33.

Changnon, S.A., Kunkel, K.E., Reinke, B.C., 1996. Impacts and responses to the 1995 heat wave: a call to action. Bull. Am. Meteorol. Soc. 77, 1497-1506.

Chen, L., Fu, B., Zhao, W., 2008. Source-sink landscape theory and its ecological significance. Front. Biol. China 3, 131-136.

Chen, L., Tian, H., Fu, B., Zhao, X., 2009. Development of a new index for integrating landscape patterns with ecological processes at watershed scale. Chin. Geogr. Sci. 19, 37-45.

Chen, A., Yao, L., Sun, R., Chen, L., 2014. How many metrics are required to identify the effects of the landscape pattern on land surface temperature? Ecol. Indic. 45, 424-433.

Connors, J.P., Galletti, C.S., Chow, W.T., 2013. Landscape configuration and urban heat island effects: assessing the relationship between landscape characteristics and land surface temperature in Phoenix, Arizona. Landscape Ecol. 28, 271-283.

Cushman, S.A., McGarigal, K., Neel, M.C., 2008. Parsimony in landscape metrics: strength, universality, and consistency. Ecol. Indic. 8, 691-703.

EPA, 2009. Urban Heat Island Mitigation (accessed on 11.3.2014) http://www.epa. gov/hiri/mitigation/index.htm.

Fischer, E., Seneviratne, S., L̈1thi, D., Sch r, C., 2007. Contribution of land-atmosphere coupling to recent European summer heat waves. Geophys. Res. Lett. 34, L06707. 
Frumkin, H., McMichael, A., 2008. Climate change and public health thinking, communicating, acting. Am. J. Prev. Med. 35, 403-410.

Gastwirth, J.L., 1972. The estimation of the Lorenz curve and Gini index. Rev. Econ. Stat. 54, 306-316.

Imhoff, M.L., Zhang, P., Wolfe, R.E., Bounoua, L., 2010. Remote sensing of the urban heat island effect across biomes in the continental USA. Remote Sens. Environ. $114,504-513$.

Jiménez-Muñoz, J.C., Sobrino, J., 2003. A generalized single-channel method for retrieving land surface temperature from remote sensing data. J. Geophys. Res. 108, 4688-4695.

Ku‘ulei, S.R., Kido, M.H., Jokiel, P.L., Edmonds, T., Brown, E.K., 2012. Use of integrated landscape indicators to evaluate the health of linked watersheds and coral reef environments in the Hawaiian Islands. Environ. Manage. 50, $21-30$.

Kupfer, J.A., 2012. Landscape ecology and biogeography Rethinking landscape metrics in a post-FRAGSTATS landscape. Prog. Phys. Geogr. 36, 400-420.

Li, J., Song, C., Cao, L., Zhu, F., Meng, X., Wu, J., 2011. Impacts of landscape structure on surface urban heat islands: a case study of Shanghai, China. Remote Sens. Environ. 115, 3249-3263.

Li, X., Zhou, W., Ouyang, Z., Xu, W., Zheng, H., 2012. Spatial pattern of greenspace affects land surface temperature: evidence from the heavily urbanized Beijing metropolitan area, China. Landscape Ecol. 27, 887-898.

Li, Z.-L., Tang, B.-H., Wu, H., Ren, H., Yan, G., Wan, Z., Trigo, I.F., Sobrino, J.A., 2013. Satellite-derived land surface temperature: current status and perspectives. Remote Sens. Environ. 131, 14-37.

Li, X., Li, W., Middel, A., Harlan, S.L., Brazel, A.J., Turner Ii, B.L., 2016. Remote sensing of the surface urban heat island and land architecture in Phoenix, Arizona: combined effects of land composition and configuration and cadastral-demographic-economic factors. Remote Sens. Environ. 174 233-243.

Liu, H., Weng, Q., 2009. An examination of the effect of landscape pattern, land surface temperature, and socioeconomic conditions on WNV dissemination in Chicago. Environ. Monit. Assess. 159, 143-161.

Liu, D., Li, Y., Kong, F., 2013. Spatial distribution of land surface temperature in central city proper and the cooling of objects effect: a case study of Nanjing. Remote Sens. Land Resour. 25, 117-122 (In Chinese with English Abstract).

McGarigal, K., Marks, B.J., 1994. Fragstats. Spatial pattern analysis program for quantifying landscape structure. Version 2.

McGarigal, K., Ene, E., Holmes, C., Cushman, S., Neel, M., 2002. FRAGSTATS: Spatial Pattern Analysis Program for Categorical Maps.

McGarigal, K., Tagil, S., Cushman, S., 2009. Surface metrics: an alternative to patch metrics for the quantification of landscape structure. Landscape Ecol. 24, 433-450.

McGarigal, K., Cushman, S., Ene, E., 2012. FRAGSTATS v4: Spatial Pattern Analysis Program for Categorical and Continuous Maps. Computer software program produced by the authors at the University of Massachusetts, Amherst.

Mirzaei, P.A., Haghighat, F., 2010. Approaches to study urban heat island-abilities and limitations. Build. Environ. 45, 2192-2201.
Nichol, J.E., Fung, W.Y., Lam K.-s. Wong, M.S., 2009. Urban heat island diagnosis using ASTER satellite images and 'in situ' air temperature. Atmos. Res. 94 276-284.

O’neill, R., Krummel, J., Gardner, R., Sugihara, G., Jackson, B., DeAngelis, D., Milne, B., Turner, M., Zygmunt, B., Christensen, S., 1988. Indices of landscape pattern. Landscape Ecol. 1, 153-162.

Oberndorfer, E., Lundholm, J., Bass, B., Coffman, R.R., Doshi, H., Dunnett, N., Gaffin, S., Köhler, M., Liu, K.K.Y., Rowe, B., 2007. Green roofs as urban ecosystems: ecological structures, functions, and services. BioScience 57, 823-833.

Opdam, P., Luque, S., Jones, K.B., 2009. Changing landscapes to accommodate for climate change impacts: a call for landscape ecology. Landscape Ecol. 24, 715-721.

Saaroni, H., Ben-Dor, E., Bitan, A., Potchter, O., 2000. Spatial distribution and microscale characteristics of the urban heat island in Tel-Aviv, Israel. Landscape Urban Plann. 48, 1-18.

Schindler, S., von Wehrden, H., Poirazidis, K., Wrbka, T., Kati, V., 2013. Multiscale performance of landscape metrics as indicators of species richness of plants, insects and vertebrates. Ecol. Indic. 31, 41-48.

Tobler, W.R., 1970. A computer movie simulating urban growth in the Detroit region. Econ. Geogr. 46, 234-240.

Turner, M., 1989. Landscape ecology: the effect of pattern on process. Annu. Rev. Ecol. Syst. 20, 171-197.

Uuemaa, E., Mander, Ü., Marja, R., 2013. Trends in the use of landscape spatial metrics as landscape indicators: a review. Ecol. Indic. 28, 100-106.

Voogt, J.A., Oke, T.R., 2003. Thermal remote sensing of urban climates. Remote Sens. Environ. 86, 370-384.

Voogt, J.A., 2002. Urban heat island. Encycl. Global Environ. Change 3, 660-666.

Wang, J., Wang, K., Wang, P., 2007. Urban heat (or cool) island over Beijing from MODIS land surface temperature. J. Remote Sens. Beijing 11, 330.

Weng, Q., Liu, H., Lu, D., 2007. Assessing the effects of land use and land cover patterns on thermal conditions using landscape metrics in city of Indianapolis, United States. Urban Ecosyst. 10, 203-219.

Weng, Q., 2009. Thermal infrared remote sensing for urban climate and environmental studies: methods, applications, and trends. ISPRS J. Photogramm. Remote Sens. 64, 335-344.

Wu, J., 2010. Urban sustainability: an inevitable goal of landscape research. Landscape Ecol. 25, 1-4.

Yu, S., BIAN, L., LIN, X., 2005. Changes in the spatial scale of Beijing UHI and urban development. Sci. China (Ser. D Earth Sci.) 48, 97-106.

Zhou, W., Huang, G., Cadenasso, M.L., 2011. Does spatial configuration matter? Understanding the effects of land cover pattern on land surface temperature in urban landscapes. Landscape Urban Plann. 102, 54-63. 